\title{
Synthesis of Polyamides and Polyesters by Direct Polycondensation with Picryl Chloride
}

\author{
Hozumi TANAKa, Guo-chuang Wu, Yuki IWANAGA, \\ Kohei SANUI, and Naoya OGATA \\ Department of Chemistry, Sophia University, \\ 7-1 Kioi-cho, Chiyoda-ku, Tokyo 102, Japan.
}

(Received December 10, 1981)

\begin{abstract}
KEY WORDS Direct Polycondensation / Polyamide / Polyester / Picryl Chloride / Pyridine / Aminobenzoic Acid / 2,5-Pyridinedicarboxylic Acid / Terephthalic Acid /
\end{abstract}

Polycondensation reactions of bifunctional monomers are generally carried out at elevated temperatures above $200^{\circ} \mathrm{C}$ since the reactions are usually accompanied by equilibrium between the esterification or the amidation and the hydrolysis. Enhancement of monomer reactivity can be achieved by using active monomers such as acid chloride so that polycondensation reactions take place under mild conditions.

On the other hand, various methods have been developed to obtain polyamides or polyesters di-

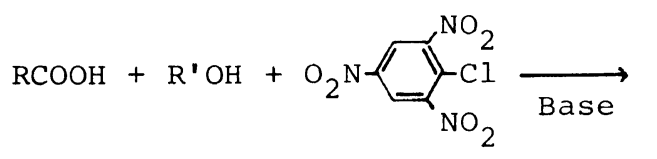

This reaction was applied to the direct polycondensation of dicarboxylic acid and diol or diamine, and it was found that polyamides and polyesters rectly from dicarboxylic acid and diamines or diols under mild conditions by direct polycondensation with phosphorylation. ${ }^{1-7}$

This paper deals with direct polycondensation without the use of phosphorylation reactions. Recently, it was found ${ }^{8}$ that a condensation reaction takes place at room temperature between acid and alcohol to form almost quantitatively an ester compound in organic solvents in the presence of picryl chloride and a base.

could be obtained very rapidly at room temperature by the following reaction,

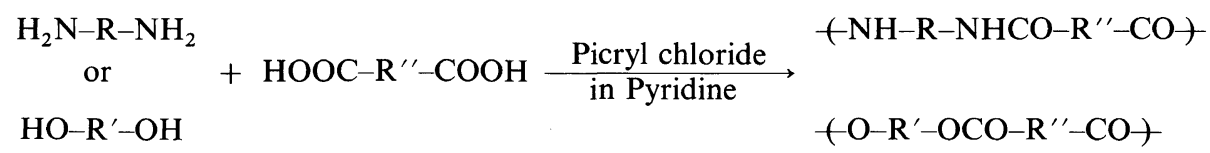

The general procedure used for the synthesis of polyamide from $p$-aminobenzoic acid is as follows: $0.004 \mathrm{~mol}$ of $p$-aminobenzoic acid was dissolved in $10 \mathrm{~cm}^{3}$ of pyridine and then $0.005 \mathrm{~mol}$ of picryl chloride was added to the solution with stirring at room temperature. As soon as the picryl chloride was added, an exothermic reaction took place im- mediately and the solution became heterogeneous with a separation of a solid polymer. After a given period of time, excess acetone was added to remove the solvent and by-product from the polymer, which was then isolated by filtration. The polymer was washed repeatedly with water and acetone, and dried under reduced pressure. The polyester was 
H. TANAKA et al.

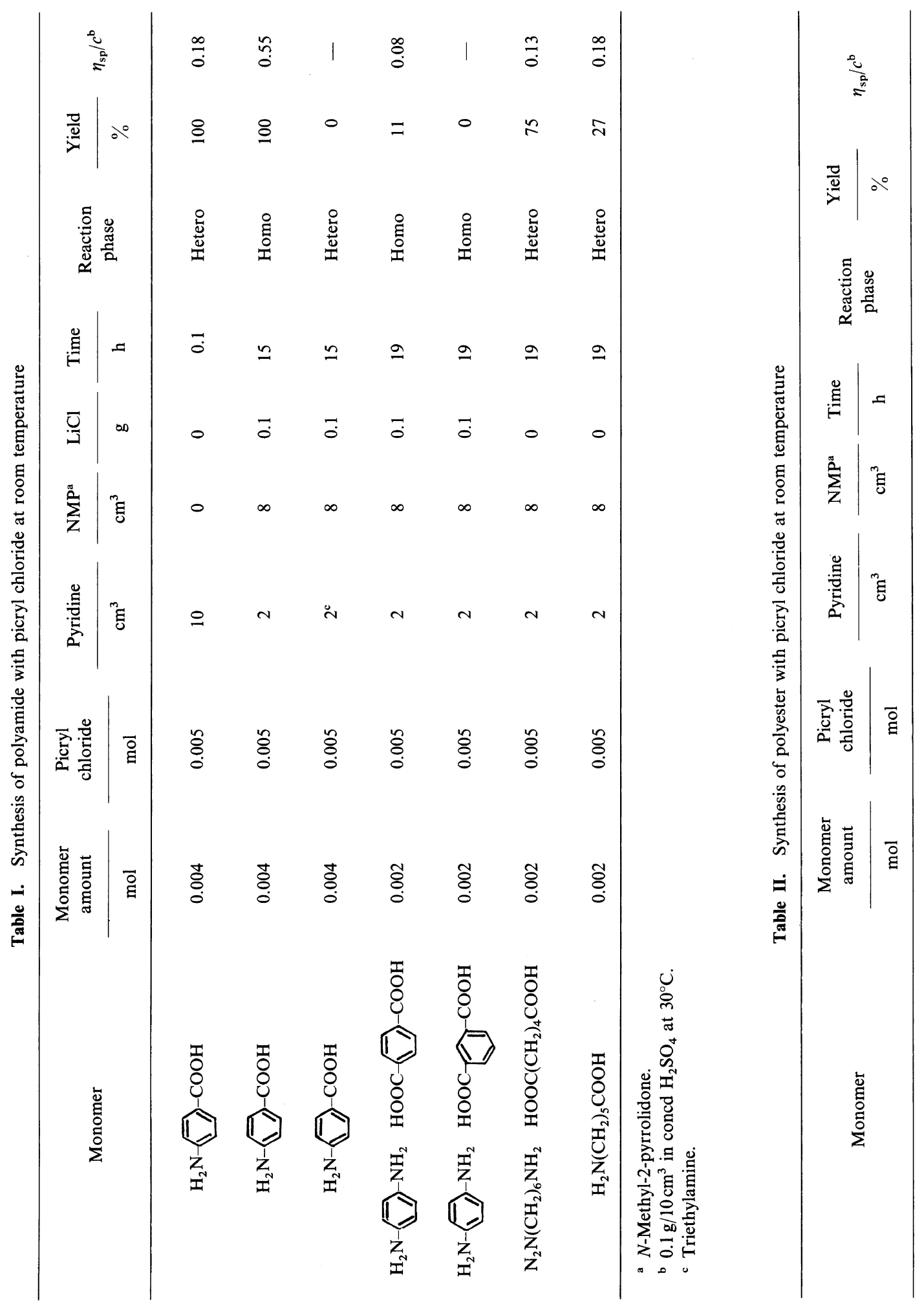


Synthesis of Polyamides and Polyesters

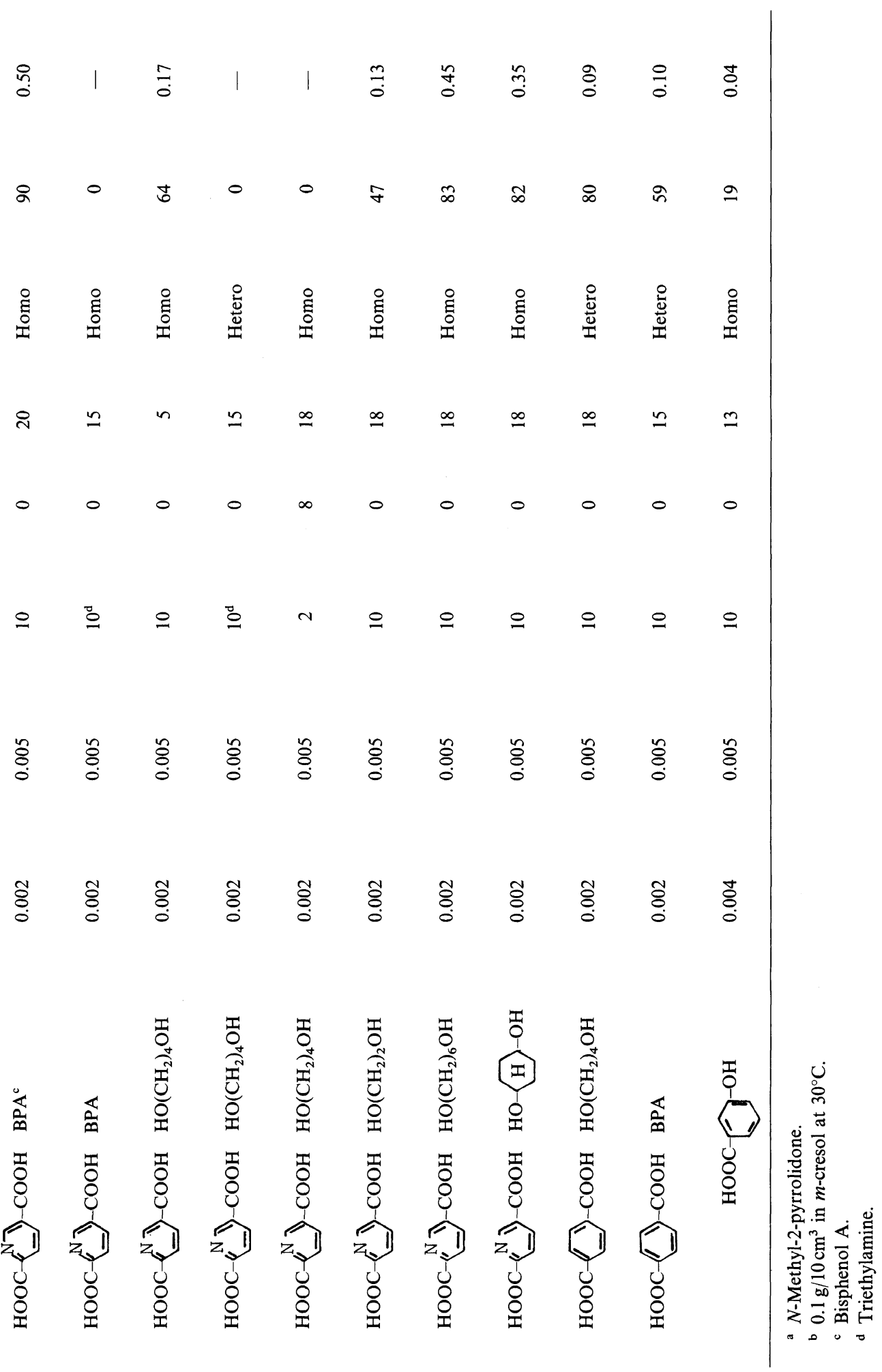


obtained by a method similar to this. The solution viscosities of polyamides and polyesters were determined in concentrated sulfuric acid and $m$-cresol at $30^{\circ} \mathrm{C}$, respectively. Table I summarizes the results of the polycondensation of various monomers in the presence of picryl chloride, where it was seen that polyamides were obtained in a good yield from $p$ aminobenzoic acid, and a combination of aromatic carboxylic acid and diamine failed to give satisfactory results in terms of both polymer yield and solution viscosity. When triethylamine was used in place of pyridine, no polymer was obtained.

The polycondensation of aliphatic carboxylic acid and diamine resulted in a higher yield than that of an $\omega$-amino acid such as $\varepsilon$-amonocaproic acid. Table II shows the results of the synthesis of polyesters from various dicarboxylic acids and diols. Bisphenol A (BPA) and 2,5-pyridinedicarboxylic acid (2,5-PyDC) gave polyesters with relatively high solution viscosities in a pyridine solution, but no polymer was obtained in a triethylamine solution.

1,6-Hexanediol gave a polyester with a higher solution viscosity than those from ethyleneglycol and 2,5-pyridinedicarboxylic acid. Terephthalic acid and 1,4-butanediol yielded a much lower molecular weight polyester than that from 2,5-PyDC.

Since neither polyamide nor polyester could be obtained in a triethylamine solution, it is thought that pyridine might possibly participate with picryl chloride in the reaction.

A possible reaction mechanism is suggested by the following,

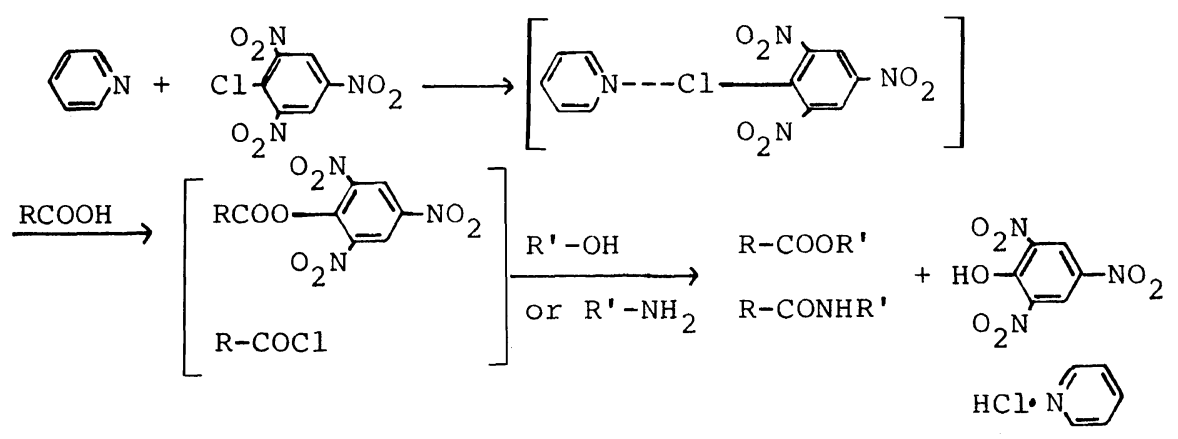

Additional detailed studies on direct polycondensation in polyamide and polyester syntheses are being carried out so as to determine the optimum reaction conditions and reaction mechanism involved in this process. The results will be published in the near future.

\section{REFERENCES}

1. N. Ogata and H. Tanaka, Polym. J., 2, 672 (1971).

2. N. Ogata and H. Tanaka, Polym. J., 3, 365 (1973).
3. N. Ogata and H. Tanaka, Polym. J., 6, 461 (1974).

4. N. Ogata, K. Sanui, and M. Harada, J. Polym. Sci., Polym. Chem. Ed., 17, 2401 (1979).

5. N. Yamazaki, F. Higashi, and J. Kawabata, $J$. Polym. Sci., Polym. Chem. Ed., 12, 2149 (1974).

6. G. Wu, H. Tanaka, K. Sanui, and N. Ogata, $J$. Polym. Sci., Polym. Lett. Ed., 19, 343 (1981).

7. N. Ogata, K. Sanui, H. Tanaka, and S. Yasuda, Polym. J., 13, 989 (1981).

8. S. Takimoto, J. Inanaga, T. Katsuki, and $\mathbf{M}$. Yamaguchi, the National Meeting of the Chemical Society of Japan, Preprint, 33, 908 (1975). 